\title{
Pathologic Study of Supernumerary Orbital Band in Type I Duane Syndrome
}

\author{
Muhammad Hassaan Ali ${ }^{a}$ Stacy L. Pineles ${ }^{a}$ Federico G. Velez ${ }^{a, c, d} \quad$ Anika K. Tandon ${ }^{a, b}$ Ben J. Glasgow ${ }^{a}$ \\ aStein Eye Institute, Departments of Ophthalmology and Pathology and Laboratory Medicine, UCLA, Los Angeles, CA, USA; \\ ${ }^{b}$ Doheny Eye Institute, Department of Ophthalmology, UCLA, Los Angeles, CA, USA; ' Department of Ophthalmology, Weill \\ Cornell Medical College, New York, NY, USA; ${ }^{\mathrm{d}}$ Department of Ophthalmology, Duke University, Durham, NC, USA
}

\section{Established Facts}

- Accessory orbital bands are a rare finding and have been reported to produce strabismus.

- Most of the bands are labeled as fibrous or muscular on the basis of radiological evidence.

\section{Novel Insights}

- Accessory orbital bands that are predominantly fibrous seem to produce strabismus.

- Compressed collagen may appear red using Masson trichrome staining. In such cases, identification may require polarization, Sirius red staining, and immunohistochemistry.

\section{Keywords}

Supernumerary band - Accessory orbital band . Histopathology · Strabismus · Duane syndrome

\begin{abstract}
Background/Aims: Accessory orbital bands are relatively rare and very few reports detail histopathology. Cases in the literature describe the composition of the bands as muscular and/ or fibrous. The composition of the supernumerary band lying deep in the medial rectus muscle in a patient with type I Duane syndrome was investigated. Methods: Histochemical stains were used in conjunction with polarized light for differ-
\end{abstract}

entiating compressed collagen from muscle. Immunohistochemistry was used for verification of the presence of muscle. Results: Compressed collagen appeared red using Masson trichrome staining. Collagen was positively identified by illumination with polarized light on several stains including the underutilized Sirius red dye. Conclusions: The findings of dense collagen fibers in the fibrotic band with focal striated muscle correlated with the restrictive strabismus. In concert with other cases in the literature, it is proposed that the fibrous bands are generally associated with restrictive strabismus. Bands that are muscular may or may not be associated with strabismus. Special techniques are needed to positively identify compressed collagen.

\section{KARGER}

(c) 2019 S. Karger AG, Basel

E-Mail karger@karger.com

www.karger.com/oop
Ben Glasgow, MD

Stein Eye Institute, Department of Ophthalmology, UCLA

100 Stein Plaza, Los Angeles, CA 90095 (USA)

E-Mail bglasgow@mednet.ucla.edu 


\section{Introduction}

Accessory bands are rare orbital structures associated with restrictive strabismus. The bands are usually described as an incidental intraoperative finding or with the use of high-resolution orbital imaging [1]. Very few reports have described detailed histopathology of these supernumerary bands. The histopathology may provide insight into possible mechanisms for strabismus in such cases [2,3]. Fibrosis following injury is preceded or accompanied by inflammatory infiltrates. We here present a case of restrictive strabismus due to an accessory band in Duane syndrome that underwent detailed histopathological examination. The findings have implications for the approach to strabismus in these patients.

\section{Case Report}

The subject was a 35-year-old man with symptomatic diplopia and strabismus. He had previously been diagnosed with esotropia (ET) secondary to type I left Duane syndrome and had no history of previous ocular surgery or trauma. His past medical, family and social histories were noncontributory.

On ophthalmic examination, best corrected visual acuity was $20 / 20$ and 20/50 in the right and left eye, respectively. Stereopsis measured $40 \mathrm{~s}$ of arc at near with a significant left head turn. A marked limitation to abduction with narrowing of the palpebral fissure was observed in the left eye. Full ocular motility was observed in the right eye. Prisms demonstrated $20 \Delta$ of left (ET) and $2 \Delta$ of left hypotropia in the primary position, $6 \Delta$ of left ET in up gaze and $20 \Delta$ of left ET in down gaze. ET significantly increased in left gaze. He was orthotropic in right gaze. Slit lamp and dilated fundus examinations were unremarkable in both eyes.

The patient underwent preoperative forced duction testing. Severe restriction to abduction was apparent in the left eye. A paralimbal conjunctival incision was made. An extremely tight medial rectus muscle was found inserted $5.5 \mathrm{~mm}$ posterior to the limbus. A spring-loaded muscle clamp was placed on the medial rectus muscle and the muscle was disinserted from the sclera. A 6-0 polyglactin 910 suture was secured on the margin of the medial rectus muscle. Prior to reinserting the muscle, forced duction testing was repeated after removal of the eyelid speculum. Severe restriction to abduction persisted, despite complete disinsertion of the medial rectus muscle. Careful inspection of the sclera revealed an accessory band, distinct from the retracted muscle (Fig. 1). The band maintained a separate insertion, approximately $2 \mathrm{~mm}$ posterior to the muscle insertion and extended deep into the orbit. Following detachment and excision of this band, the forced duction test showed no remaining restriction in abduction. The left medial rectus muscle was recessed $4.5 \mathrm{~mm}$ posterior to its insertion. Attention then shifted to the right eye. The right medial rectus muscle was recessed 5.5 $\mathrm{mm}$ and the lateral rectus muscle was recessed $6.5 \mathrm{~mm}$ with an adjustable suture technique.
Histopathologic Studies

The accessory band was fixed in formalin and processed for paraffin embedment. Step sections were taken for histopathologic study including hematoxylin and eosin, Masson trichrome stain, elastic Masson trichrome, and Sirius red stains as previously described [46]. Polarizing microscopy was performed on stained sections to further distinguish collagen from smooth muscle. Immunohistochemistry for reactivity to anti-muscle-specific actin was used to verify the presence or absence of muscle. Appropriate tissues served as positive and negative controls. In addition, deletion of the primary antibody served as an additional negative control.

\section{Results}

Sections stained with hematoxylin and eosin of the accessory band revealed closely packed fibers that showed birefringence on polarization. In one focal area, striated muscle was seen with faint cross-striations and peripheral nuclei (Fig. 2). However, most of the tissue appeared to be compressed fibrous tissue. The Masson trichrome stain showed marked red staining but also birefringence with polarized light (Fig. 3a, b). To clarify this apparent disparity, Sirius red staining was performed (Fig. 4a). Staining was markedly enhanced in most areas where the Masson trichrome appeared red. These areas demonstrated birefringence and dichroism with polarized light further supporting the presence of collagen (Fig. 4b). Immunohistochemistry for muscle-specific actin was performed and showed only small areas of reactivity in the section (Fig. 5).

\section{Discussion/Conclusion}

The key findings of this study are the evidence that supernumerary bands that induce strabismus are composed mainly of fibrous tissue. Further histological studies of fibrous tissue that are under tension should not rely solely on Masson trichrome as there may be a false-positive red staining for muscle.

The term "supernumerary band" is largely applied to most accessory, soft tissue structures in the orbit. The clinically published cases of supernumerary bands without histopathology mainly describe the atypical muscular bands; these are most commonly identified in the superonasal orbit [7-10]. However, our study shows mainly fibrous tissue in the band with exiguous striated and smooth muscle. Only 6 prior studies detail histopathology (Table 1). In a cadaver study from an apparently asymptomatic individual with accessory bands, the bands 


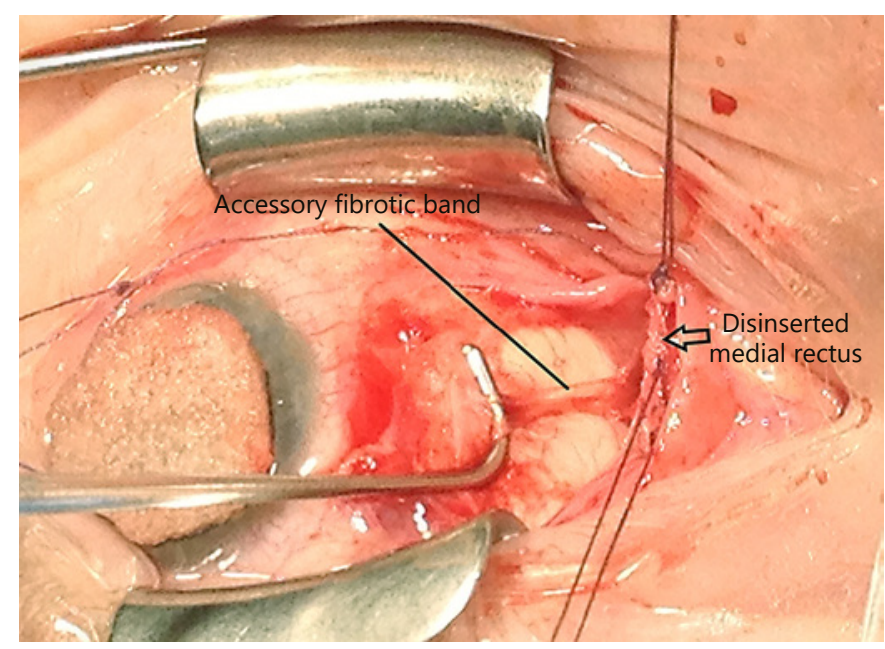

Fig. 1. Perioperative finding of an accessory orbital band.

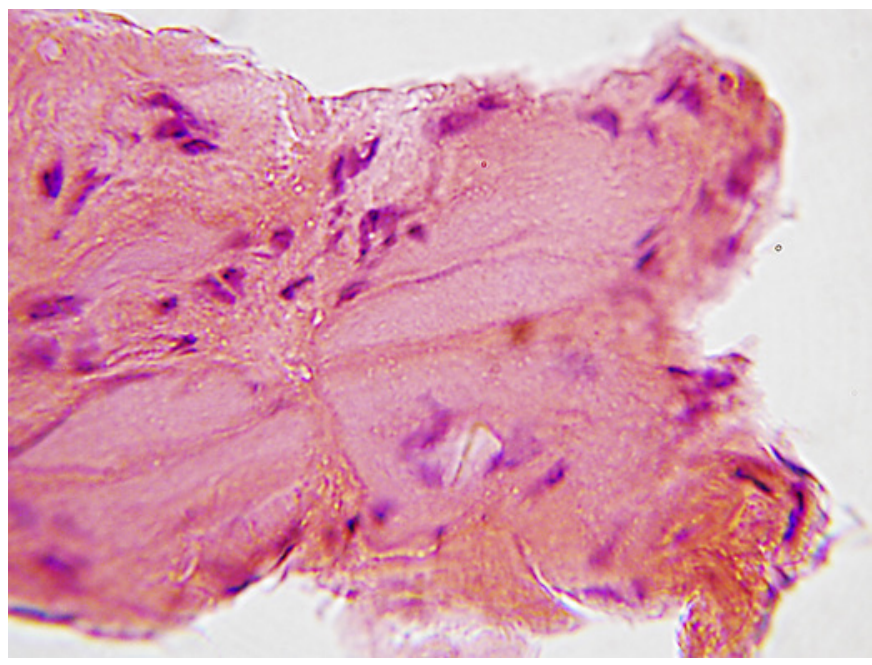

Fig. 2. Photomicrograph of the focal striated muscle in the resected band specimen. Hematoxylin and eosin.
Fig. 3. a Photomicrograph of a section shows predominant red staining fibers with the Masson trichrome stain. b Polarization of the trichrome-stained sections highlights an irregular fibrous architecture with birefringence in areas staining red.
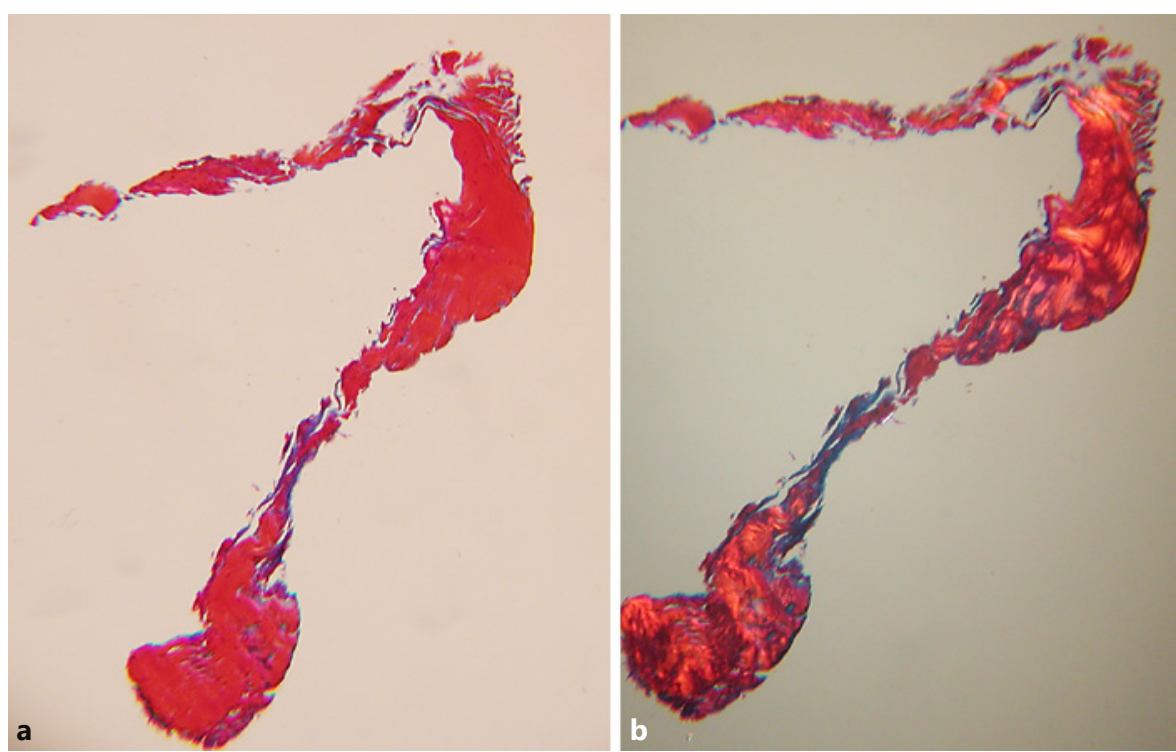

were described as muscle slips fusing the superior and inferior rectus muscles. Histological examination showed a predominance of striated muscle fibers that were well organized [11]. Similarly, another study reported bilateral accessory muscles in a patient with Gorlin syndrome with large-angle exotropia [12]. In one of the bands, histopathology presumably showed striated muscle (images not provided in the paper). This band was described as taut. Resection of this band, without resecting the contralateral band, resulted in release and in correction of the exotropia. The remaining 3 cases with histopathology suggest findings similar to our own. There was predominant fibrosis with only fragments of atrophied muscle in some instances $[2,3,13]$.

To understand the composition of the supernumerary bands, it is imperative to distinguish fibrous tissue 

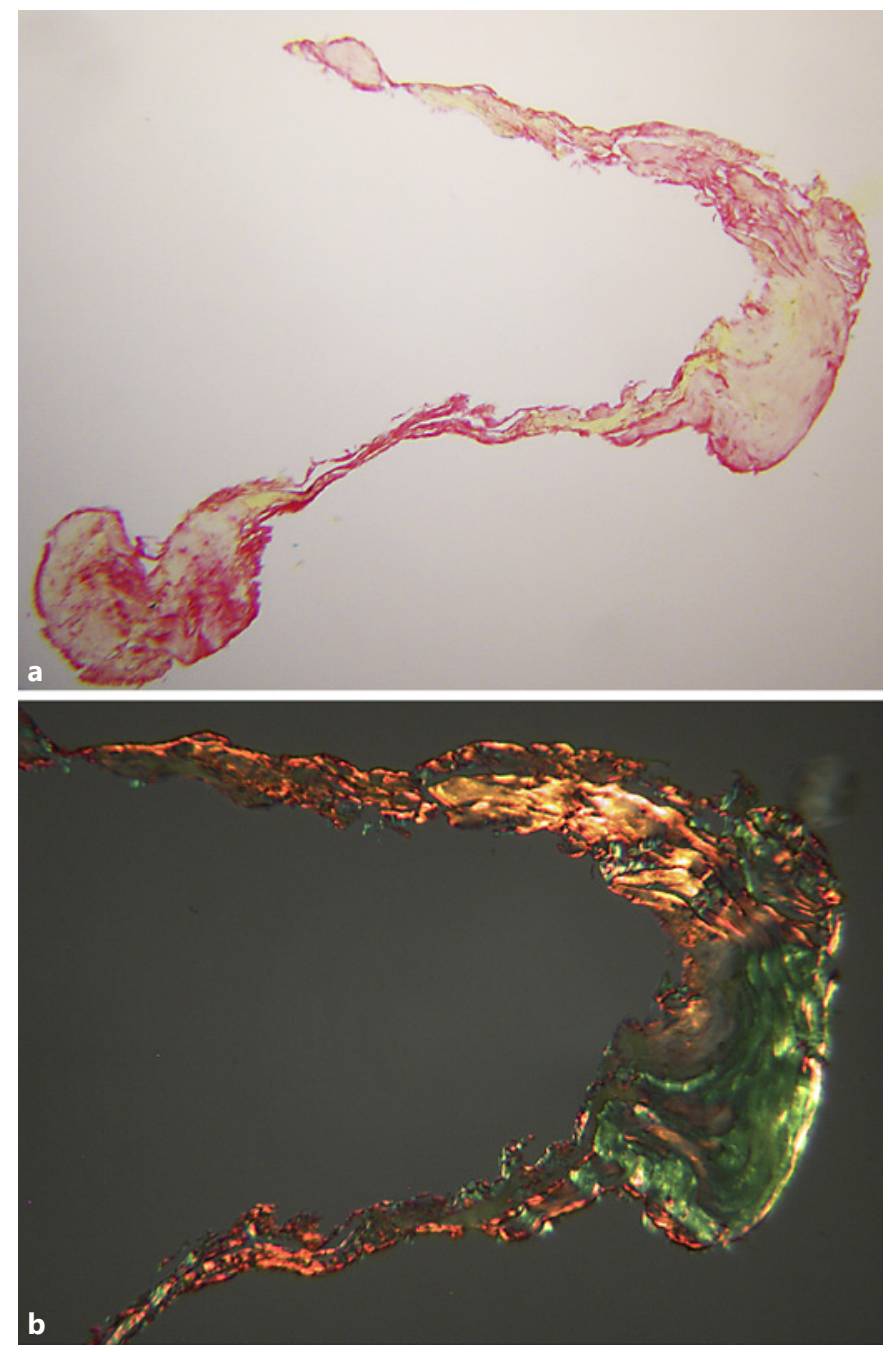

Fig. 4. Photomicrograph of a section stained with Sirius red. a The sample shows both red and poorly stained regions. b Polarized light reveals the birefringence of the red-stained areas as well as dichroism in less well-stained areas.

from muscle. One case in the literature presumed the bands were fibrous on the basis of the hematoxylin and eosin stain [2]. Others have used a trichrome stain to distinguish collagen from muscle although the images were not provided $[12,13]$. Clinically appearing muscular bands were proven to be fibrous tissue in at least one case [3]. The other cases with strabismus are variably described as fibrous and muscular, but histopathology was not performed (Table 1). The dense collagen in our study yielded a marked false-positive reaction that stained fibrous tissue red and might have been misinterpreted as muscle. The basis for the false red color in tri-

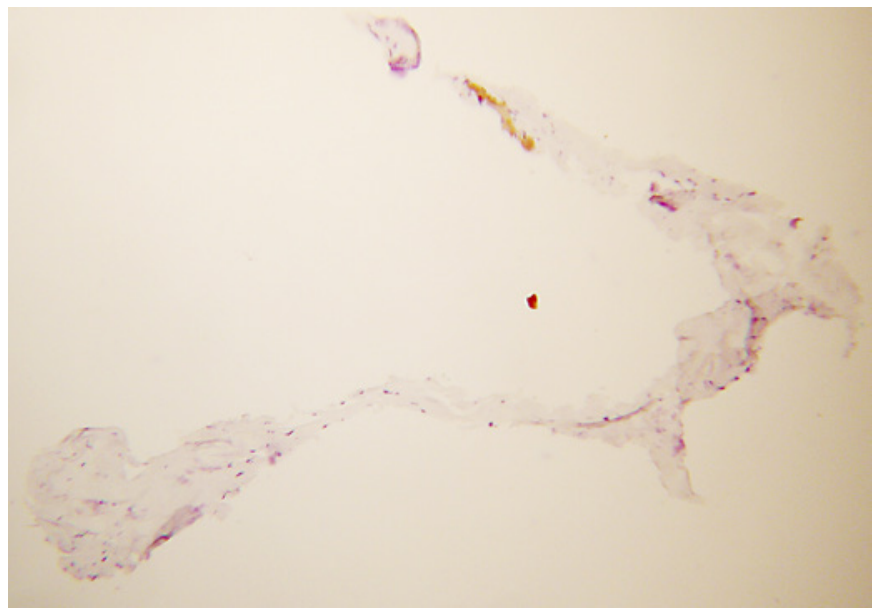

Fig. 5. Photomicrograph of immunohistochemistry with antibody to muscle-specific antigen shows only focal reactive areas. Most regions that correspond to red staining with the trichrome stain are negative for reactivity. The areas of reactivity matched the areas of striated muscle depicted on hematoxylin and eosin.

chrome staining lies in differential removal of red and blue dyes by polyacids based on the size of the stain molecules. Because collagen is porous, the red stain (Biebrich scarlet-acid fuchsin) is removed by polyacids and replaced by aniline blue. Muscle tissue is not well penetrated by the polyacids and remains red. However, in our case, the collagen stained red because the red dye was not displaced. We attribute this to the compressed nature of the fibrous tissue in the band that also accounted for its tough clinical appearance. Ocular pathologists frequently examine specimens for presumed slipped muscle and need a reliable stain to differentiate dense fibrous tissue from muscle. Sirius red is an excellent alternative histochemical stain that has excellent inter-observer reliability and reproducibility in renal specimens [4-6]. In our study, polarization-induced birefringence in both the hematoxylin and eosin and Masson trichrome stain alerted us that red areas may actually be collagen. This was confirmed in Sirius red stains. Polarization microscopy also enhanced the birefringent substructure of collagen in the Sirius red stain and showed dichroism in areas that poorly stained for Sirius red.

The clinical counterpart of the compressed collagen fibers in the accessory band may be the severe restrictive strabismus and progressive tightness of the medial rectus muscle. The key to diagnosis was the intraoperative examination and repeat forced duction testing. Prior to the ini- 


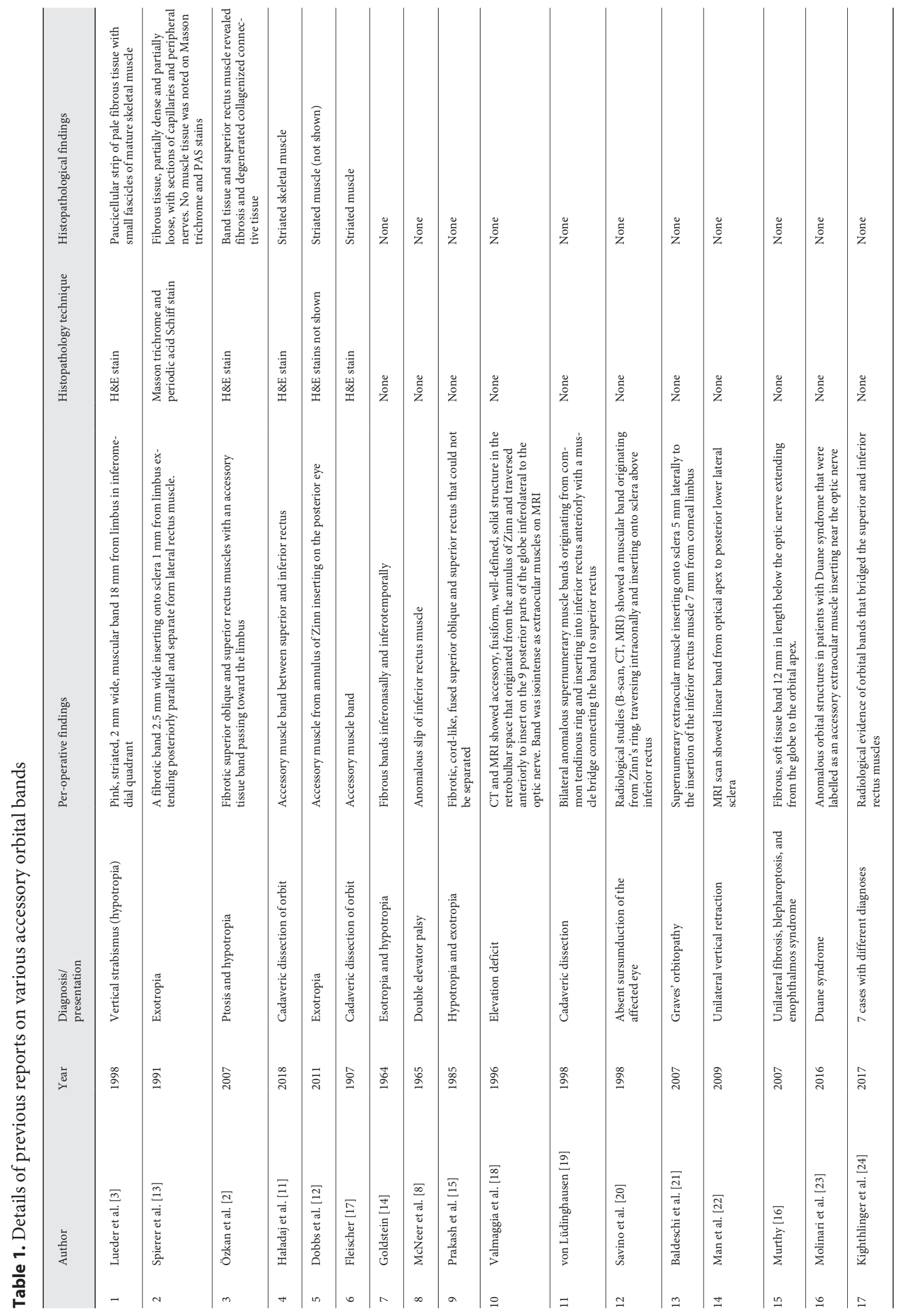


tial conjunctival incision, the restricted eye had a positive forced duction test. After disinsertion of the medial rectus muscle, the tough band was clearly visualized and repeat forced duction test was again positive. It was not until the accessory band was disinserted that the eye moved freely. The clinical findings match the pathologic findings of predominant fibrous tissue and analysis of the special stains reveal some challenges with interpretation of stains.

Khitri and Demer [1] imaged accessory bands using high-resolution surface coil magnetic resonance imaging. They reported two cases with type III Duane syndrome that had bands superonasally along the levator complex. Because the bands were inaccessible deep in the orbit, they were not amenable to excision and histopathology. Some reports suggest the presence of accessory fibrous tissue bands but do not provide detailed histopathology $[14,15]$.

The histopathology of the cases of accessory bands may provide some mechanistic insight. For those patients with histopathology, the history suggests a congenital onset of strabismus. These patients invariably show no evidence inflammation of the muscle (Table 1). The fibrosis does not appear to result from a focal insult but rather a disorder of mesodermal development. Early diagnosis of accessory orbital bands is clinically challenging and sometimes not achievable; however, in patients with atypical strabismus, it is paramount to broaden the differential diagnosis to include these structures. Orbital imaging can considerably facilitate preoperative identification and allow for timely surgical planning $[1,13,16]$. Another telltale finding of supernumerary bands is the "traction-indention" sign. When attempting to rotate the eye against restriction, the atypical band may produce significant opposition, causing localized pressure with secondary scleral indentation [2]. However, in patients with atypical restrictive strabismus and a supernumerary band, disinsertion of both the accessory tissue and weakening of the associated extraocular muscle is often required.
In conclusion, we emphasize the importance of careful peroperative examination and forced duction testing in cases of restrictive strabismus. A positive forced duction could possibly be caused by an accessory orbital band which should be excised to relieve the restriction. Our review indicates that to date the pathologically verified cases of fibrotic supernumerary bands had all been associated with strabismus, most characteristically hypotropia. However, strabismus may be caused by either muscular or fibrous bands. Histopathological studies with appropriate stains for collagen and the judicious use of polarization microscopy are helpful to elicit the composition of these accessory bands. The clinical history in concert with the histopathology suggest fibrosis of supernumerary bands occurring at birth implying a developmental rather than an acquired origin.

\section{Statement of Ethics}

The authors have no ethical conflicts to disclose.

\section{Disclosure Statement}

The authors have no conflicts of interest to declare.

\section{Funding Sources}

The authors have not received any funding.

\section{Author Contributions}

M.H.A.: Manuscript writing, final critical review. S.L.P.: Data collection, final critical review. F.G.V.: Data collection, final critical review. A.K.T.: Data collection, final critical review.

B.J.G.: Manuscript writing, data analysis, final critical review.

\section{References}

1 Khitri MR, Demer JL. Magnetic resonance imaging of tissues compatible with supernumerary extraocular muscles. Am J Ophthalmol. 2010 Dec;150(6):925-31.

2 Özkan SB, Çakmak H, Dayanir V. Fibrotic superior oblique and superior rectus muscles with an accessory tissue band. J AAPOS. 2007 Oct;11(5):491-4.

3 Lueder GT, Dunbar JA, Soltau JB, Lee BCP, McDermott M. Vertical strabismus resulting from an anomalous extraocular muscle. J AAPOS. 1998;2(2):126-8.
4 Farris AB, Adams CD, Brousaides N, Della Pelle PA, Collins AB, Moradi E, et al. Morphometric and visual evaluation of fibrosis in renal biopsies. J Am Soc Nephrol. 2011 Jan; 22(1):176-86.

5 Grimm PC, Nickerson P, Gough J, McKenna R, Jeffery J, Birk P, et al. Quantitation of allograft fibrosis and chronic allograft nephropathy. Pediatr Transplant. 1999 Nov;3(4):257-70.
6 Grimm PC, Nickerson P, Gough J, McKenna $\mathrm{R}$, Stern E, Jeffery J, et al. Computerized image analysis of Sirius Red-stained renal allograft biopsies as a surrogate marker to predict longterm allograft function. J Am Soc Nephrol. 2003 Jun;14(6):1662-8.

7 Lueder GT. Anomalous orbital structures resulting in unusual strabismus. Surv Ophthalmol. 2002 Jan-Feb;47(1):27-35. 
8 McNeer KW, Jampolsky A. Double elevator palsy: caused by anomalous insertion of the inferior rectus. Am J Ophthalmol. 1965 Feb; 59(2):317-9.

9 Sacks JG. The levator-trochlear muscle. A supernumerary orbital structure. Arch Ophthalmol. 1985 Apr;103(4):540-1.

10 Wylen EL, Brown MS, Rich LS, Hesse RJ. Supernumerary orbital muscle in congenital eyelid retraction. Ophthal Plast Reconstr Surg. 2001 Mar;17(2):120-2.

11 Haładaj R, Wysiadecki G, Polguj M, Topol M Bilateral muscular slips between superior and inferior rectus muscles: case report with discussion on classification of accessory rectus muscles within the orbit. Surg Radiol Anat. 2018 Jul;40(7):855-62.

12 Dobbs MD, Mawn LA, Donahue SP. Anomalous Extraocular Muscles with Strabismus. AJNR Am J Neuroradiol. 2011 Oct 1;32 (9):E167-8.

13 Spierer A, Desatnik H, Barishak YR. Aberrant extraocular fibrous band as a cause of strabismus. Am J Ophthalmol. 1991 Mar;111(3): $370-1$.
14 Goldstein JH. The intraoperative forced duction test: report of a case of congenital fibrous bands. Arch Ophthalmol. 1964 Nov;72(5): 647-9.

15 Prakash P, Menon V, Ghosh G. Congenital fibrosis of superior rectus and superior oblique: a case report. Br J Ophthalmol. 1985 Jan;69(1):57-9.

16 Murthy R. Unilateral restrictive ophthalmoplegia and enophthalmos associated with an intraorbital tissue band. J AAPOS. 2007 Dec; 11(6):626-7.

17 Fleischer B. Musculus retractor bulbi und drittes Lid bei einer menschlichen Missbildung. Anat Anz. 1907;30:465-70.

18 Valmaggia C, Zaunbauer W, Gottlob I. Elevation deficit caused by accessory extraocular muscle. Am J Ophthalmol. 1996 Apr;121(4): 444-5.

19 von Lüdinghausen M. Bilateral supernumerary rectus muscles of the orbit. Clin Anat. 1998;11(4):271-7.
20 Savino G, D'Ambrosio A, Tamburrelli C, Colosimo C, Dickmann A. Restrictive limitation of sursumduction caused by an anomalous muscular structure. Ophthalmologica. 1998; 212(6):424-8.

21 Baldeschi L, Bisschop PH, Wiersinga WM. Supernumerary extraocular muscle in Graves' orbitopathy. Thyroid. 2007 May;17(5):479-80.

22 Man F, Wang Z, Wang J, Zhang F, Jiao Y. Unilateral vertical retraction syndrome with orbital band. J AAPOS. 2009 Aug;13(4): 419-21.

23 Molinari A, Plager D, Merino P, Galan MM, Swaminathan M, Ramasuramanian S, et al. Accessory extraocular muscle as a cause of restrictive strabismus. Strabismus. 2016 Dec; 24(4):178-83.

24 Kightlinger BS, Saraf-Lavi E, Sidani C. Anomalous Extraocular Muscles: A Case Series of Orbital Bands Connecting the Superior Rectus to Inferior Rectus. Neurographics. 2017; $7(2): 88-91$. 